\title{
Glycyrrhizin improves the pathogenesis of psoriasis through IL-17A and the SIRT1-STAT3 axis
}

\author{
Huang Qiong \\ Huashan Hospital \\ Ling Han \\ Huashan Hospital \\ Nan Zhang \\ Huashan Hospital \\ Hu Chen \\ Huashan Hospital \\ Ke Yan \\ Huashan Hospital \\ Zheng Zhang \\ Huashan Hospital \\ Ying Ma ( $\nabla$ yingmayingma@126.com ) \\ Huashan Hospital \\ Jin Xu \\ Huashan Hospital
}

\section{Research Article}

Keywords: Psoriasis, glycyrrhizin, IL-17A, SIRT1-STAT3 pathway

Posted Date: December 16th, 2020

DOI: https://doi.org/10.21203/rs.3.rs-111541/v1

License: (c) (1) This work is licensed under a Creative Commons Attribution 4.0 International License.

Read Full License 


\section{Abstract}

Background: The anti-inflammatory effect of glycyrrhizin has been widely recognized, while the specific mechanism of glycyrrhizin in psoriasis remains poorly understood.

Results: Here, in an imiquimd-induced mouse model of psoriasis (IMD), we found that glycyrrhizin can substantially improve the adverse symptoms in mice. The hematoxylin-eosin staining results showed that glycyrrhizin can also improve the pathological state of skin cells in IMD mice. Using enzyme-linked immunosorbent assay (ELISA), we found that glycyrrhizin substantially inhibited the expression of IL-17A and IFN- $\gamma$ in the serum of IMD mice. In order to simulate the effect of IL-17A on keratinocytes in psoriasis, we treated HaCaT cells with $100 \mathrm{ng} / \mathrm{mL}$ IL-17A (IL-17A-HaCaT cells) for $48 \mathrm{~h}$. Then, using cell-counting kit-8 (CCK-8) and ELISA assays, we found that glycyrrhizin inhibited the proliferation of IL-17A-HaCaT cells and reversed the promotion of IL-6, CCL20, and TNF-a induced by IL-17A. Further, western blotting (WB) results indicated that glycyrrhizin promoted the expression of SIRT1 and inhibited the expression of STAT3 and phosphorylated STAT3 (p-STAT3). By treating IL-17A-HaCaT cells with EX-527 (a potent and selective inhibitor of SIRT1), combined with CCK-8 and WB experiments, we initially found that EX-527 inhibited the proliferation of IL-17A-HaCaT cells and promoted the expression of STAT3, p-STAT3, and acetylated STAT3 (a-STAT3). However, when glycyrrhizin was added at the same time, the proliferation of IL-17A-HaCaT cells increased, and the expression of STAT3, p-STAT3, and a-STAT3 reduced. We then knocked down the expression of SIRT1 via small interfering RNA in IL-17A-HaCaT cells, and the results were consistent with those of EX-527.

Conclusions: Together, these results indicated that glycyrrhizin improved psoriasis by inhibiting the expression of IL-17A and IFN- $\gamma$ in vivo and suppressed the proliferation of IL-17A-HaCaT cells and the expression of STAT3, p-STAT3, and a-STAT3 by upregulating SIRT1 in vitro.

\section{Highlight}

1. Glycyrrhizin improves psoriasis by reducing the secretion of IL-17A and FIN- $\gamma$ in serum.

2. Glycyrrhizin may inhibit the proliferation of keratinocytes by reducing the expression of IL-17A and IFN-y.

3. Glycyrrhizin may inhibit the proliferation of keratinocytes through the SIRT1-STAT3 pathway.

4. Glycyrrhizin inhibits the expression of p-STAT3 and a-STAT3 through a SIRT1-dependent pathway

\section{Introduction}

Clinically, glycyrrhizin is often used to treat patients with acute and chronic hepatitis, liver poisoning, and early liver cirrhosis [1, 2], and the efficacy of glycyrrhizin in improving psoriasis has attracted the attention of clinicians. A previous study showed that glycyrrhizin combined with methotrexate (MTX) can be used as an effective alternative therapy for the treatment of erythrodermic psoriasis with bullous pemphigoid [3]. Moreover, glycyrrhizin combined with acitretin can improve psoriasis by regulating T helper 17 (TH17) 
cells [3]. However, the clinical value of glycyrrhizin in psoriasis has not been fully developed; hence, it is necessary to further study its mechanism to provide a new strategy for the clinical treatment of psoriasis.

Psoriasis is a complex disease caused by an imbalance in the dynamic interaction of immune cells, keratinocytes, endothelial cells, and other cells, and these cells play important roles in the initial and maintenance stages of psoriasis [4]. And psoriasis is considered as the chronic T helper 1(TH1)/TH17mediated inflammatory disease [5]. IL-17A is mainly synthesized and secreted by TH17 cells [6] and is considered to play a key role in the pathogenesis of psoriasis $[7,8]$. Moreover, IL-17A is also key to the interaction between $\mathrm{TH} 17$ cells and keratinocytes. Studies have shown that the expression of many cytokines and chemokines, such as TNF-a, IL-6, IL-17A, and CCL20, in keratinocytes were stimulated by IL17A secreted by TH17 cells $[9,10]$. Therefore, we aimed to further explore the relationships between glycyrrhizin, TH17 cells, and keratinocytes.

The imbalance between pro-inflammatory and anti-inflammatory signals may lead to the development of psoriasis [12]. Keratinocytes are the main source of inhibitory cytokines, which maintains the skin in an inflammatory quiescent state [4]. A previous study has shown that SIRT1 inhibits keratinocyte proliferation and promotes keratinocyte differentiation [11]. Therefore, SIRT1 may regulate the release of inhibitory cytokines by affecting the keratinocyte proliferation and play a key role in the occurrence and development of psoriasis.

A previous study reported that oxidative stress contributes to the pathogenesis of psoriasis, and the activation of SIRT1 inhibits the MAPK, NF-KB, and STAT3 oxidative stress signaling pathways, thereby down-regulating inflammatory factors and inhibiting excessive keratinocyte proliferation [13]. The activation of STAT3 in keratinocytes partly stimulates the activation of Langerhans cells through IL-1a, and their presence is vital to the pathogenesis of psoriasis through the production of IL-23 [12]. Therefore, we speculate that the SIRT1-STAT3 axis may play a crucial role in glycyrrhizin regulation during the pathogenesis of psoriasis, which is worthy of further study.

Based on the above findings, our study sheds light on the following important connections between glycyrrhizin, IL-17A, SIRT1, STAT3, and the pathogenesis of psoriasis. First, we set out to characterize the regulatory relationship between glycyrrhizin and IL-17A in vivo. We found that glycyrrhizin inhibits the expression of IL-17A and its related genes, IL-6, CCL20, and TNF- $a$. In addition, the regulatory relationship between glycyrrhizin and the SIRT1-STAT3 axis was investigated in vitro, and the results showed that glycyrrhizin inhibits STAT3 expression by promoting SIRT1 expression. In summary, these data indicate that glycyrrhizin may play a therapeutic role in improving psoriasis through IL-17A and the SIRT1-STAT3 axis.

\section{Materials And Methods \\ Clinical Samples}


Clinical skin samples from patients with psoriasis and healthy persons (same age and sex as patients) were collected from Huashan Hospital (Shanghai, China). The experiment was approved by the Huashan Hospital Clinical Research Ethics Committee. All methods were carried out in accordance with relevant guidelines and regulations, and all participants/donors provided written informed consent for the study.

\section{Animal Experiments}

All male BALB/c mice (10 weeks old, 25-35 g) were obtained from the Animal Experiment Centre of Huashan Hospital and fed under sterile specific-pathogen-free (SPF) conditions. The mice were randomly assigned into four groups ( $n=3$ each) for further study, and the mice in group A were not treated. In groups B-D, mice were evenly smeared with $62.5 \mathrm{mg}$ imiquimod cream on their backs once a day for three consecutive days. On the fourth day, $50 \mathrm{mg}$ imiquimod cream was applied evenly on the backs of the mice once a day for two consecutive days. On day seven, the psoriasis animal models (imiquimd-induced mouse model of psoriasis, IMD) were successfully established in the B-D groups. In group B, normal saline was intragastrical injected for the positive control group (model group). MTX has been used to treat psoriasis and other skin diseases for more than 50 years [13]. For mice in group C, 20 mg/kg MTX was intragastrical injected for the treatment control group (model + MTX group). In addition, in mice of group D, $20 \mathrm{mg} / \mathrm{kg}$ glycyrrhizin was intragastrical injected for the treatment observation group (model + glycyrrhizin group). In groups B-D, the treatment was performed once a day for four consecutive days, and on the eleventh day, the back skin of the mice in groups A-D was photographed, and the mice were sacrificed by cervical dislocation. The animal experiments used meet the requirements of animal welfare and animal ethics of Huashan Hospital, and are approved by the Ethics Committee of animal experiments of Huashan Hospital.

\section{Cell culture and treatment}

$\mathrm{HaCaT}$ cells were purchased from the cell bank of the Chinese Academy of Sciences and met the cell line STR identification criteria. HaCaT cells were cultured in Dulbecco's Modified Eagle's Medium (L110, Basamedia Biology, Shanghai, China) supplemented with 10\% fetal bovine serum (Thermo Fisher), 100 $\mathrm{g} / \mathrm{mL}$ penicillin, and $100 \mathrm{~g} / \mathrm{mL}$ streptomycin (L110, Basamedia Biology, Shanghai, China) in a moist incubator with $5 \% \mathrm{CO} 2$ at $37^{\circ} \mathrm{C}$. Wild type HaCaT cells were treated with IL-17A (100 ng/mL), glycyrrhizin $(2 \mu \mathrm{M})$, and IL-17A $(100 \mathrm{ng} / \mathrm{mL})+$ glycyrrhizin $(2 \mu \mathrm{M})$ for $48 \mathrm{~h}$. The IL-17A-HaCaT cells (treated with 100 $\mathrm{ng} / \mathrm{mL}$ IL-17A) were treated with glycyrrhizin (2 $\mathrm{MM}$ ) and EX527 (100 nM, SIRT1 inhibitor, Med Chem Express) for $48 \mathrm{~h}$.

\section{Cell-counting kit-8 (CCK-8)}

$\mathrm{HaCaT}$ cells were seeded in a 96 -well plate at a density of $5 \times 10^{3}$ cells per well (triple replication). The next day, after the HaCaT cells adhered, the culture solution was discarded, and a mixture of $10 \mu \mathrm{L}$ CCK-8 solution $+90 \mu \mathrm{L}$ serum-free medium was added, and the cells were cultured in an incubator for $2 \mathrm{~h}$. After $2 \mathrm{~h}$, the absorbance of the cells at $450 \mathrm{~nm}$ was measured using a microplate reader (SpectraMax M2e; Molecular Devices, Sunnyvale, CA, USA). The above steps were repeated for five consecutive days. 


\section{Immunohistochemical experiments}

For the immunohistochemical analyses, skin samples were dewaxed and rehydrated, followed by endogenous peroxidase quenching, antigen retrieval (saline sodium citrate, autoclaving), and blocking with $10 \%$ goat serum. The sections were then incubated with anti-rabbit STAT3 antibody (1:2000, CST, USA), phosphorylated STAT3 (p-STAT3) antibody (1:2000, CST, USA), acetylated STAT3 (a-STAT3) antibody (1:2000, CST, USA), SIRT1 antibody (1:2000, CST, USA), AMPK antibody (1:2000, CST, USA), phosphorylated AMPK (p-AMPK) antibody (1:2000, CST, USA), and a-tublin antibody (1:2000, CST, USA). After washing with PBS, the sections were incubated for 50 min with secondary antibody, stained with DAB working solution, and then counterstained with hematoxylin.

\section{Enzyme linked immunosorbent assay (ELISA)}

The cell lysis solution was collected to detect the expression of IL-6 (1:2000, Abcam, USA), TNF-a (1:2000, Abcam, USA), and CCL20 (1:2000, Abcam, USA) according to the manufacturer' s instructions. The absorbance was then measured with a microplate reader (SpectraMax M2e, USA) at $450 \mathrm{~nm}$.

\section{Real-time fluorescence quantitative PCR (RT-PCR)}

Real-time fluorescence quantitative PCR (RT-PCR)

Trizol reagent (Thermo Fisher Scientific) was used to extract total RNA from cells and tissues, and a reverse transcription kit (TaKaRa) was used to reverse the total RNA to cDNA. The primers used in this experiment were listed in Table 1. The fluorescent quantitative PCR kit (TaKaRa) was used to perform RTPCR and 2- $\triangle \triangle C T$ was used to calculate the relative gene expression. a-tublin was used as an internal reference.

\section{Western blotting}

The protein concentrations were determined using the bicinchoninic acid protein assay kit (Beyotime, China). All protein concentrations were adjusted to $2 \mathrm{mg} / \mathrm{mL}$, and then separated by $8 \%$ SDS-PAGE. Then, the proteins were transferred to a nitrocellulose membrane (731809, Millipore, USA) and blocked with $5 \%$ skimmed milk for $1 \mathrm{~h}$, and the membrane was sequentially incubated with a primary antibody and a horseradish peroxidase-conjugated secondary antibody. Finally, the enhanced chemiluminescence method was used to detect protein levels.

\section{siRNA knockdown of SIRT1 expression}

$\mathrm{HaCaT}$ cells in the logarithmic phase were incubated in six-well plates at a density of $5 \times 10^{4}$ cells $/ \mathrm{mL}$ overnight. The next day, HaCaT cells were transfected with siRNAs (Table 2) using Lipofectamine 3000 (L3000015, Thermo Fisher Scientific), and incubated for $24 \mathrm{~h}$ in accordance with the manufacturer's instructions. Then, fresh media was added, and the corresponding stimulant was added to the media for $48 \mathrm{~h}$. 


\section{Statistical analysis}

Data are presented as the mean \pm S.D. of at least three independent experiments. One-way analysis of variance was performed using SPSS 19.0 software (SPSS Inc., Chicago, IL, USA) to determine the statistical significance of the results. $P<0.05$ was considered statistically significant.

\section{Results}

\section{Glycyrrhizin can improve the adverse symptoms of psoriasis in IMD}

As shown in Fig. 1A (upper part), on the seventh day, the obvious clinical symptoms of psoriatic lesions appeared on the back skin of IMD mice, specifically as keratinocyte hyperproliferation, erythema, scales, etc. However, after four consecutive days of treatment with MTX or glycyrrhizin, the adverse symptoms of IMD mice substantially improved (Fig. 1A, lower part). Furthermore, the HE staining showed obvious keratinization, thickened spinous cells, spine extension and fluctuations, and inflammatory cell infiltration in the skin cells of IMD mice that lacked treatment on day 11, while these pathological phenomena substantially improved in the skin cells of MTX- or glycyrrhizin-treated IMD mice (Fig. 1B). In order to further investigate whether the potential mechanism of glycyrrhizin for the treatment of psoriasis involves the regulation of the inflammatory response, ELISA was used to detect the expression of IL-17A and IFN- $Y$ in the serum of wild-type mice or IMD mice, with or without treatment. The results showed that IL-17A and IFN- $y$ expression in the serum of IMD mice treated with MTX or glycyrrhizin was substantially reduced compared to expression levels in non-treated mice (Fig. 1C). Together, glycyrrhizin may improve the clinical symptoms and pathological status of IMD by inhibiting the expression of IL-17A and IFN- $\gamma$ in the serum.

Glycyrrhizin inhibits the proliferation of HaCaT cells and reverses the high expression of IL-6 and CCL20 induced by IL-17A

The CCK-8 results showed that glycyrrhizin substantially inhibited the proliferation of HaCaT cells and reversed the increase in the proliferation of HaCaT cells caused by IL-17A (Fig. 2A). In addition, as shown in Fig. 2B, the expression of IL-6 and CCL20 substantially increased in the lysate and supernatant of IL$17 \mathrm{~A}-\mathrm{HaCaT}$ cells. However, when glycyrrhizin was added, glycyrrhizin substantially reversed the increased expression of IL-6 (Fig. 2B) and CCL20 (Fig. 2C) induced by IL-17A. Moreover, the ELISA results showed that glycyrrhizin substantially reduced the expression of TNF- $a$ in the lysate and supernatant of $\mathrm{HaCaT}$ cells and effectively reversed TNF-a stimulation by IL-17A (Fig. 2D). Together, glycyrrhizin suppresses keratinocyte proliferation and the expression of inflammatory factors related to IL-17A in HaCaT cells.

\section{Glycyrrhizin promotes SIRT1 expression and reduces STAT3 expression}

The mRNA expression of STAT3 was up-regulated while SIRT1 was down-regulated in human psoriasis skin tissues than normal skin tissues via RT-PCR detection (Figure 3A). Compared with the skin tissues of 
IMD model, the protein expression of STAT3 was down-regulated while SIRT1 was up-regulated in Model+MTX or Model+GA group via western blotting detection (Figure 3B). The immunohistochemistry results showed that compared with the skin cells of normal subjects, the skin cells of patients with psoriasis showed substantially increased STAT3 expression and substantially decreased SIRT1 expression (Figure 3C). When treated with glycyrrhizin, STAT3 expression decreased and SIRT1 expression increased in IMD (Figure 3D). Moreover, glycyrrhizin substantially reversed the high expression of STAT3 and p-STAT3 and reversed the low expression of SIRT1 induced by IL-17A, at the protein level, in $\mathrm{HaCaT}$ cells (Figure 4A and B). Together, these results indicate that glycyrrhizin may improve psoriasis by promoting SIRT1 expression and inhibiting STAT3 expression.

\section{Glycyrrhizin suppresses keratinocyte proliferation and STAT3 expression by upregulating SIRT1}

As shown in Figure 5A, EX-527 promoted the proliferation of IL-17A-HaCaT cells, while glycyrrhizin substantially reduced this effect. This finding suggests that glycyrrhizin substantially reversed the effect of SIRT1 on the proliferation of keratinocytes. Further, western blotting experiments showed that EX-527 substantially promoted the expression of STAT3, p-STAT3, and a-STAT3 in IL-17A-HaCaT cells. When glycyrrhizin was added at the same time, the expression of STAT3, p-STAT3, and a-STAT3 increased upon EX-527 treatment (Figure 5B and C). Moreover, interfering RNAs (siRNA-1, siRNA-2, and siRNA-3) that knock down SIRT1 were constructed, and the RT-PCR results showed that siRNA-1 substantially knocked down SIRT1 expression in IL-17A-HaCaT cells (Figure 6A). As shown in Figure 6B, the CCK-8 results further confirmed that reduced SIRT1 expression promoted keratinocyte proliferation, and glycyrrhizin substantially inhibited the effect of SIRT1 on cell proliferation. In addition, the western blotting results indicated that when IL-17A-HaCaT were treated with glycyrrhizin, the STAT3, p-STAT3, and a-STAT3 expression levels that increased upon SIRT1 knockdown were substantially reversed (Figure 6C and D). Together, these results suggest that glycyrrhizin inhibits keratinocyte proliferation and promotes keratinocyte differentiation by promoting SIRT1 expression and inhibiting STAT3 expression, thereby playing a therapeutic role in psoriasis.

\section{Discussion}

In this study, we characterized the biological role of glycyrrhizin in psoriasis in vivo and in vitro. Glycyrrhizin is a therapeutic substance that improves psoriasis by down-regulating IL-17A and INF- $y$, and further inhibits the expression of IL-6, TNF-a, and CCL20 induced by IL-17A. Mechanically, glycyrrhizin inhibits the expression of STAT3 by reversing the inhibitory effect of IL-17A on SIRT1, which may be a potential mechanism by which glycyrrhizin improves psoriasis.

Psoriasis is a chronic immune skin disease mediated by T cells, keratinocytes, dendritic cells, and other immune cells. The interaction between IL-17A secreted by TH17 cells and epidermal keratinocytes plays a key pathogenic role in triggering psoriasis [14]. It has been reported that glycyrrhizin inhibits liver fibrosis by regulating the balance of Th1 / Th2 and TH17 / regulatory T cells in mouse models of liver fibrosis [15]. Moreover, glycyrrhizin combined with acitretin has been shown to inhibit the synergistic effects 
produced by TH17 cells, and improve psoriasis [3]. In this study, we found that glycyrrhizin can improve the adverse reactions and pathological status of IMD mice by reducing the serum expression of IL-17A, which suggests that glycyrrhizin may inhibit the secretion of IL-17A in TH17 cells and reduce the effects of IL-17A on other cells.

Increased levels of IL-17A will produce a self-amplifying inflammatory response in keratinocytes and promote keratinocyte proliferation, which will further promote the formation of mature psoriatic plaques [15]. In this study, the CCK-8 results indicated that glycyrrhizin substantially inhibited the proliferation of IL-17A-HaCaT cells. In addition to IL-17A, IFN- $y$ can also promote keratinocyte growth [16]. Using an ELISA assay, we found that glycyrrhizin not only inhibits IL-17A expression, but also inhibits IFN-Y expression. Therefore, glycyrrhizin might inhibit the growth of keratinocytes by inhibiting the expression of IL-17A and IFN- $y$.

The expression of TNF- $a$, IL-6, and CCL20 is regulated by IL-17A $[9,10]$. We found that in the lysate and supernatant of $\mathrm{HaCaT}$ cells, glycyrrhizin significantly reversed the promotion of TNF-a, IL-6, and CCL20 induced by IL-17A. In addition to IL-17A, STAT3 has been reported to regulate the expression of TNF-a, IL6 , and CCL20 [16]. Moreover, SIRT1 is involved in regulating the expression of STAT3 in various diseases, including cancer [17], diabetic kidneys[18] and hepatic gluconeogenesis [19]. In this study, we found that glycyrrhizin inhibited the expression of STAT3 by promoting the expression of SIRT1 in keratinocytes, which suggests that glycyrrhizin may also reduce the expression of TNF-a, IL-6, and CCL20 through the SIRT1-STAT3 pathway. IL-17A activates STAT3 in keratinocytes[20], and STAT3 also promotes the transcription of IL-17A[16]. Therefore, we speculate that glycyrrhizin reduces the expression of IL-17A by inhibiting the expression of STAT3, while the low expression of IL-17A further weakens the expression of STAT3 in keratinocytes, thereby forming a positive feedback pathway, which is beneficial for improving psoriasis.

Protein modifications not only affect protein homeostasis, but can also establish new cellular functions and play important and complex roles in cell signal transduction [21]. Protein phosphorylation is an important cellular regulatory mechanism in which enzymes and receptors are activated or inactivated by phosphorylation and dephosphorylation events via protein kinases [22]. In addition, acetylation of lysine residues is also a protein modification mechanism that regulates protein activity through the function of acetyltransferases [23]. STAT3 activation also depends on post-translational modifications, phosphorylation, and acetylation [24]. A previous report indicated that sunitinib reduces imiquimodinduced psoriasis-like inflammation by inhibiting p-STAT3[25]. In this study, we found that glycyrrhizin can attenuate the promotion of p-STAT3 induced by SIRT1, which suggests that glycyrrhizin may be beneficial for improving psoriasis by inhibiting the expression of p-STAT3. Moreover, IL-22 has a pathogenic role in psoriasis, and IFN- $y$ can enhance the basic expression of a-STAT3, thereby weakening the response of keratinocytes to IL-22 [26]. In this study, we found that glycyrrhizin can attenuate the promotion of a-STAT3 induced by SIRT1, which suggests that glycyrrhizin may attenuate the response of keratinocytes to IL-22 by inhibiting the expression of a-STAT3, thereby playing a role in improving psoriasis. 
In summary, glycyrrhizin may reduce the secretion of IL-17A through the SIRT1-STAT3-IL-17A pathway in TH17 cells and keratinocytes in vivo, thereby weakening the regulation of IL-17A in other cells and improving psoriasis. Particularly, glycyrrhizin improves psoriasis by inhibiting the secretion of IL-17A and IFN- $\gamma$ in TH17 cells. Meanwhile, when the secretion of IL-17A and IFN- $\gamma$ by TH17 cells decreases, keratinocyte proliferation decreases, and this also plays a role in improving psoriasis. As for keratinocytes, glycyrrhizin may inhibit keratinocyte proliferation through the SIRT1-STAT3 pathway. In addition, through the SIRT1-dependent pathway, the inhibition of p-STAT3 and a-STAT3 expression by glycyrrhizin may also be related to the improvement of psoriasis. Together, the SIRT1-STAT3 pathway may be the key for glycyrrhizin to improve psoriasis, and this pathway may not be limited to TH17 cells and keratinocytes. Therefore, in future studies, we will elucidate whether glycyrrhizin can regulate additional cells related to psoriasis through the SIRT1-STAT3 pathway.

\section{Declarations}

\section{Ethics approval and consent to participate}

All methods were carried out in accordance with relevant guidelines and regulations, and all participants provided written informed consent for the study

\section{Consent for publication.}

All authors agree to publish this article.

\section{Availability of data and materials.}

The datasets generated and/or analysed during the current study are not publicly available due to the commercial interests of Shenzhen Jianan (Group) Co., Ltd, but are available from the corresponding author on reasonable request.

\section{Acknowledgements}

We appreciate the financial support provided by Shenzhen Jianan (Group) Co., Ltd.

\section{Funding}

The Major Research and Development Project of Innovative Drugs, Ministry of Science and Technology (2017ZX09304005) and the Health Skin and Hair Research of Chinese Medical Association - L'Oreal.

\section{Author Contributions}

$\mathrm{QH}, \mathrm{HL}, \mathrm{NXZ}, \mathrm{YM}$, and JX participated in study design; QH, HL, NXZ, HYC, KY and ZHZ conducted the experiments; QH, HL, NXZ, YM, and JX performed data analysis; QH, HL, NXZ, YM, and JX wrote the manuscript. 
Conflicts of interest

All authors declare no conflict of interest. Data and information are available and there is no dispute of interest.

\section{References}

1. Hoque R, Vodovotz $\mathrm{Y}$, Mehal $\mathrm{W}$ : Therapeutic strategies in inflammasome mediated diseases of the liver. J Hepatol 2013, 58(5):1047-1052.

2. van Rossum TG, Vulto AG, Hop WC, Schalm SW: Glycyrrhizin-induced reduction of ALT in European patients with chronic hepatitis C. Am J Gastroenterol 2001, 96(8):2432-2437.

3. Mou KH, Han D, Liu WL, Li P: Combination therapy of orally administered glycyrrhizin and UVB improved active-stage generalized vitiligo. Braz J Med Biol Res 2016, 49(8).

4. Benhadou F, Mintoff D, Del Marmol V: Psoriasis: Keratinocytes or Immune Cells - Which Is the Trigger? Dermatology 2019, 235(2):91-100.

5. Zhou F, Zhu Z, Gao J, Yang C, Wen L, Liu L, Zuo X, Zheng X, Shi Y, Zhu C et al: NFKB1 mediates Th1/Th17 activation in the pathogenesis of psoriasis. Cell Immunol 2018, 331:16-21.

6. Clark RA: Human skin in the game. Sci Transl Med 2013, 5(204):204ps213.

7. Cai Y, Shen X, Ding C, Qi C, Li K, Li X, Jala VR, Zhang HG, Wang T, Zheng J et al: Pivotal role of dermal IL-17-producing gammadelta T cells in skin inflammation. Immunity 2011, 35(4):596-610.

8. Riol-Blanco L, Ordovas-Montanes J, Perro M, Naval E, Thiriot A, Alvarez D, Paust S, Wood JN, von Andrian UH: Nociceptive sensory neurons drive interleukin-23-mediated psoriasiform skin inflammation. Nature 2014, 510(7503):157-161.

9. Harper EG, Guo C, Rizzo H, Lillis JV, Kurtz SE, Skorcheva I, Purdy D, Fitch E, lordanov M, Blauvelt A: Th17 cytokines stimulate CCL20 expression in keratinocytes in vitro and in vivo: implications for psoriasis pathogenesis. J Invest Dermatol 2009, 129(9):2175-2183.

10. Chiricozzi A, Krueger JG: IL-17 targeted therapies for psoriasis. Expert Opin Investig Drugs 2013, 22(8):993-1005.

11. Blander G, Bhimavarapu A, Mammone T, Maes D, Elliston K, Reich C, Matsui MS, Guarente L, Loureiro JJ: SIRT1 promotes differentiation of normal human keratinocytes. J Invest Dermatol 2009, 129(1):41-49.

12. Nakajima K, Kataoka S, Sato K, Takaishi M, Yamamoto M, Nakajima H, Sano S: Stat3 activation in epidermal keratinocytes induces Langerhans cell activation to form an essential circuit for psoriasis via IL-23 production. J Dermatol Sci 2019, 93(2):82-91.

13. Raaby L, Zachariae C, Ostensen M, Heickendorff L, Thielsen P, Gronbaek H, Skov L, Kyvsgaard N, Madsen JT, Heidenheim $\mathrm{M}$ et al: Methotrexate Use and Monitoring in Patients with Psoriasis: A Consensus Report Based on a Danish Expert Meeting. Acta dermato-venereologica 2017, 97(4):426432. 
14. Furue M, Furue K, Tsuji G, Nakahara T: Interleukin-17A and Keratinocytes in Psoriasis. Int J Mol Sci 2020, 21(4).

15. Yamashita T, Asano Y, Taniguchi T, Nakamura K, Saigusa R, Miura S, Toyama T, Takahashi T, Ichimura Y, Yoshizaki A et al: Glycyrrhizin Ameliorates Fibrosis, Vasculopathy, and Inflammation in Animal Models of Systemic Sclerosis. J Invest Dermatol 2017, 137(3):631-640.

16. Wu S, Rhee KJ, Albesiano E, Rabizadeh S, Wu X, Yen HR, Huso DL, Brancati FL, Wick E, McAllister F et al: A human colonic commensal promotes colon tumorigenesis via activation of $\mathrm{T}$ helper type $17 \mathrm{~T}$ cell responses. Nat Med 2009, 15(9):1016-1022.

17. Zhang S, Yang Y, Huang S, Deng C, Zhou S, Yang J, Cao Y, Xu L, Yuan Y, Yang J et al: SIRT1 inhibits gastric cancer proliferation and metastasis via STAT3/MMP-13 signaling. J Cell Physio/2019, 234(9):15395-15406.

18. Hong Q, Zhang L, Das B, Li Z, Liu B, Cai G, Chen X, Chuang PY, He JC, Lee K: Increased podocyte Sirtuin-1 function attenuates diabetic kidney injury. Kidney Int 2018, 93(6):1330-1343.

19. Xu J, Li Y, Lou M, Xia W, Liu Q, Xie G, Liu L, Liu B, Yang J, Qin M: Baicalin regulates SirT1/STAT3 pathway and restrains excessive hepatic glucose production. Pharmacol Res 2018, 136:62-73.

20. Sano S, Chan KS, Carbajal S, Clifford J, Peavey M, Kiguchi K, Itami S, Nickoloff BJ, DiGiovanni J: Stat3 links activated keratinocytes and immunocytes required for development of psoriasis in a novel transgenic mouse model. Nat Med 2005, 11(1):43-49.

21. Mijakovic I, Grangeasse C, Turgay K: Exploring the diversity of protein modifications: special bacterial phosphorylation systems. FEMS microbiology reviews 2016, 40(3):398-417.

22. Ardito F, Giuliani M, Perrone D, Troiano G, Lo Muzio L: The crucial role of protein phosphorylation in cell signaling and its use as targeted therapy (Review). International journal of molecular medicine 2017, 40(2):271-280.

23. Drazic A, Myklebust LM, Ree R, Arnesen T: The world of protein acetylation. Biochimica et biophysica acta 2016, 1864(10):1372-1401.

24. Haghikia A, Ricke-Hoch M, Stapel B, Gorst I, Hilfiker-Kleiner D: STAT3, a key regulator of cell-to-cell communication in the heart. Cardiovascular research 2014, 102(2):281-289.

25. Xian D, Song J, Yang L, Xiong X, Lai R, Zhong J: Emerging Roles of Redox-Mediated Angiogenesis and Oxidative Stress in Dermatoses. Oxidative medicine and cellular longevity 2019, 2019:2304018.

26. Sestito R, Madonna S, Scarponi C, Cianfarani F, Failla CM, Cavani A, Girolomoni G, Albanesi C: STAT3-dependent effects of IL-22 in human keratinocytes are counterregulated by sirtuin 1 through a direct inhibition of STAT3 acetylation. FASEB journal: official publication of the Federation of American Societies for Experimental Biology 2011, 25(3):916-927.

\section{Tables}

Table 1. The primers used in this experiment 


\begin{tabular}{ccc}
\hline Gene & Forward Primer $\left(5^{\prime}->\mathbf{3}^{\prime}\right)$ & Reverse Primer \\
\hline Human STAT3 & CAGCAGCTTGACACACGGTA & AAACACCAAAGTGGCATGTGA \\
Mouse STAT3 & CACCTTGGATTGAGAGTCAAGAC & AGGAATCGGCTATATTGCTGGT \\
\hline Human SIRT1 & TAGCCTTGTCAGATAAGGAAGGA & ACAGCTTCACAGTCAACTTTGT \\
\hline Mouse SIRT1 & TGATTGGCACCGATCCTCG & CCACAGCGTCATATCATCCAG \\
\hline Human $\alpha$-tublin & ACCTTAACCGCCTTATTAGCCA & CACCACGGTACAACAGGCA \\
\hline Mouse $\alpha$-tublin & CCTAAACAGGTTGATAGGCCAAA & CTCGCCTTCCACAGAATCCA \\
\hline
\end{tabular}

\section{Tabl}

2. Sequences of the siRNA oligonucleotides

\begin{tabular}{lll}
\hline Name & Sense $\left(5^{\prime}-3^{\prime}\right)$ & Antisense $\left(5^{\prime}-3^{\prime}\right)$ \\
\hline siRNA1 & CCAUCUCUCUGUCACAAAUTT & AUUUGUGACAGAGAGAUGGTT \\
siRNA2 & CCCUGUAAAGCUUUCAGAATT & UUCUGAAAGCUUUACAGGGTT \\
siRNA3 & GGAGAUGAUCAAGAGGCAATT & UUGCCUCUUGAUCAUCUCCTT \\
\hline
\end{tabular}

\section{Figures}


A
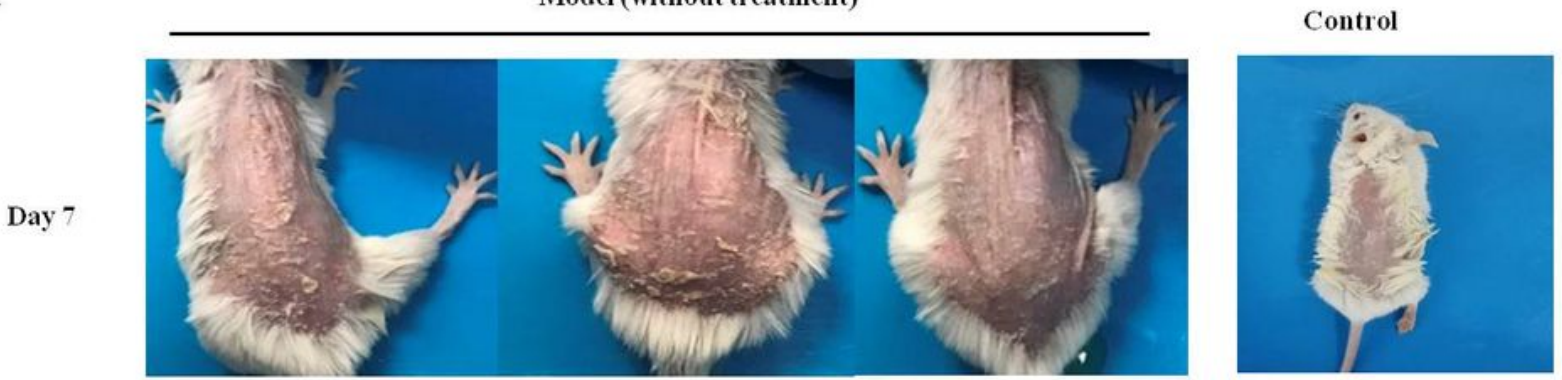

Model (with treatment)

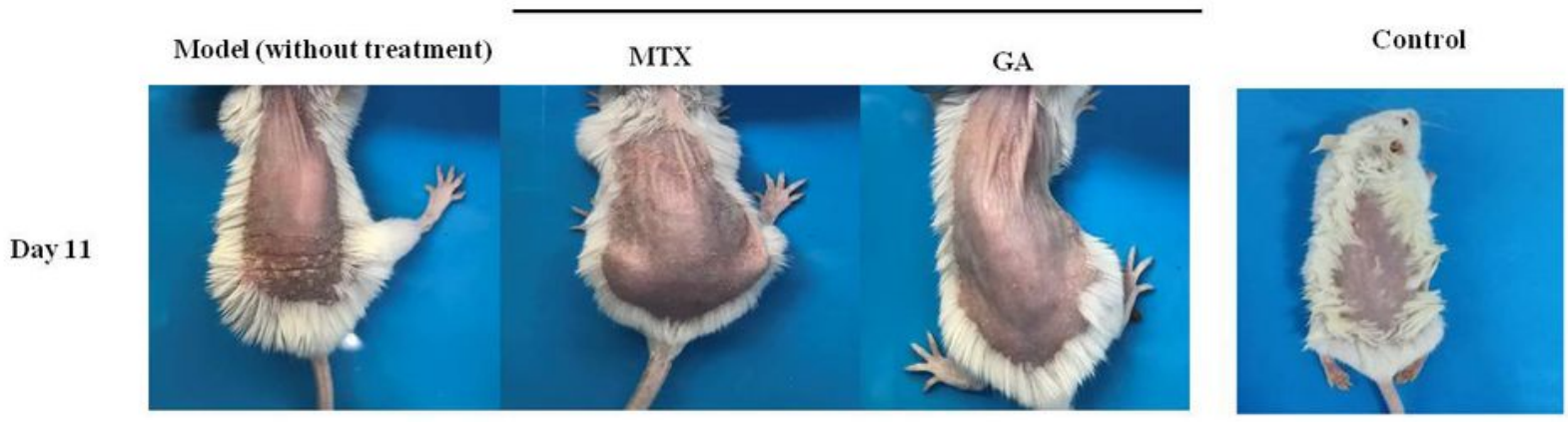

B

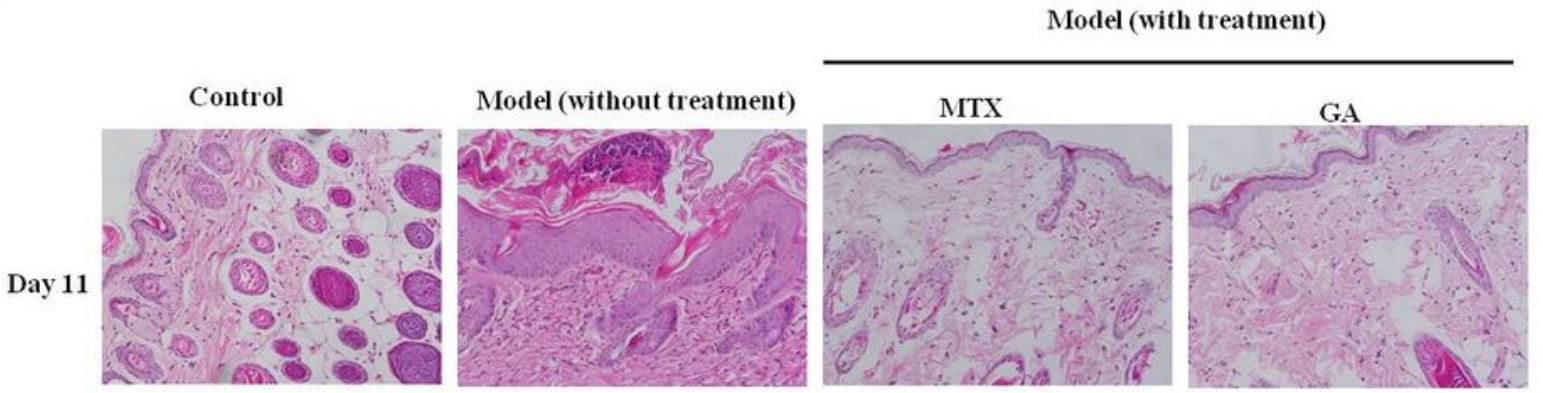

C
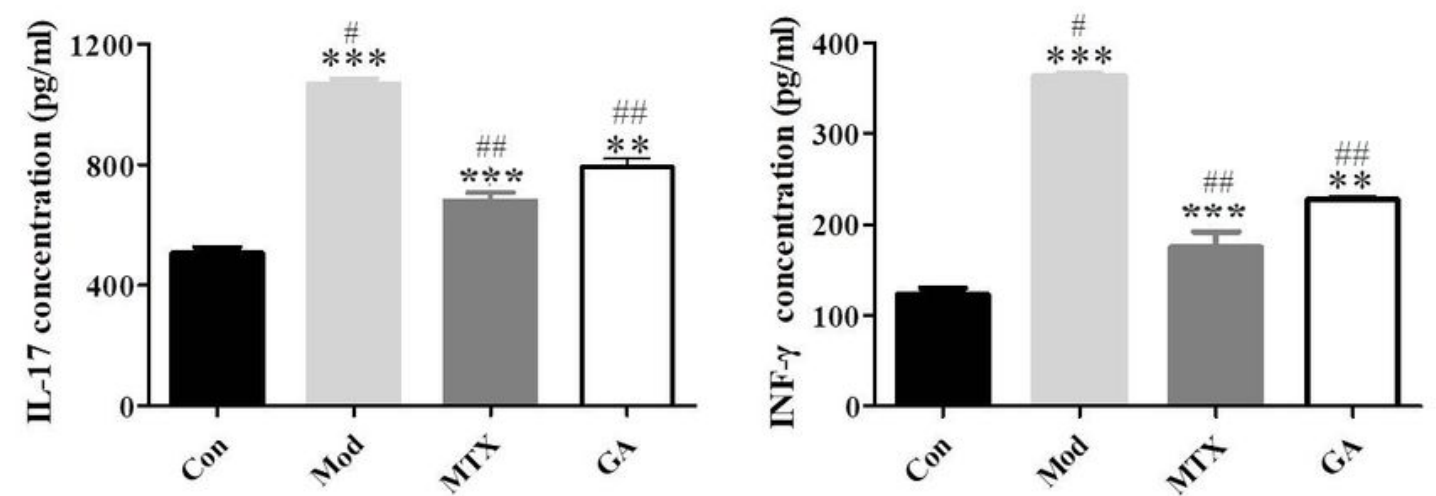

\section{Figure 1}

Glycyrrhizin may improve the adverse symptoms and pathological status of IMD by inhibiting the expression of IL-17A and IFN-y. (A) Compared with the control IMD mice (treated with normal saline), after continuous treatment with $20 \mathrm{mg} / \mathrm{kg}$ MTX or $20 \mathrm{mg} / \mathrm{kg}$ glycyrrhizin for 4 days, the adverse symptoms of IMD were substantially improved by MTX or glycyrrhizin. (B) The HE staining experiments show that MTX or glycyrrhizin substantially improved the pathological status of the skin cells in IMD. (C) 
By the ELISA experiments, we found that the pro-inflammatory cytokines IL-17A and INF-y were substantially inhibited by MTX or glycyrrhizin in the serum of IMD. NOTES: Data are presented as the mean $\pm S D(n=3) .{ }^{*} P<0.01$ and ${ }^{* * *} P<0.001 ;$ GA represents glycyrrhizin; IL-17 represents IL-17A; Mod represents IMD mice; \#compared with control group; \#\#compared with Mod group.

A

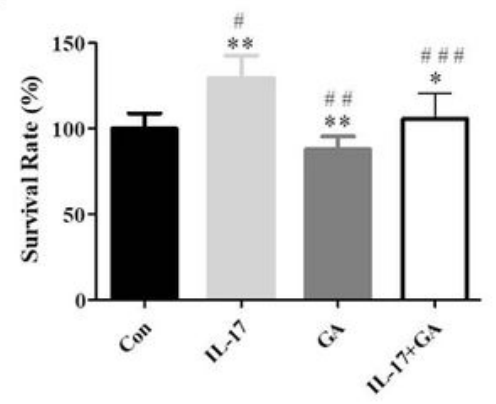

$\mathrm{B}$

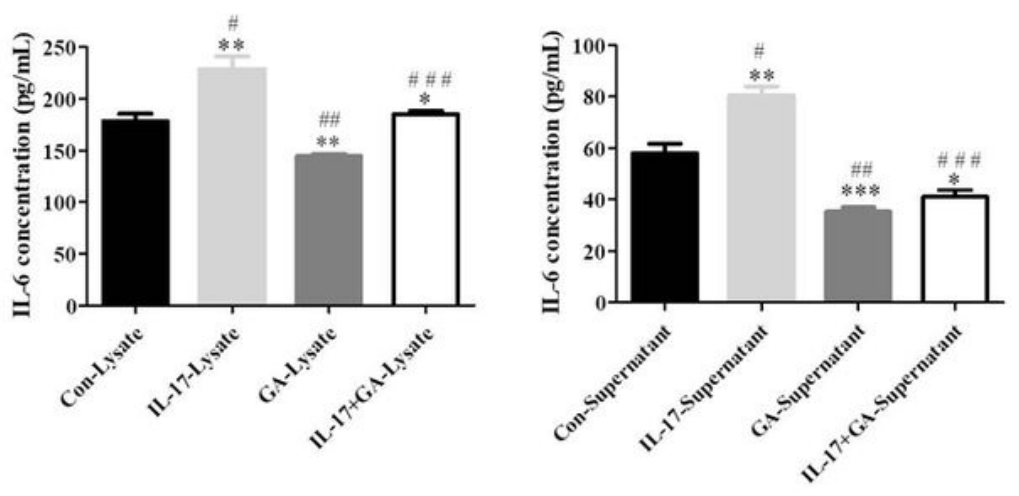

$\mathrm{C}$
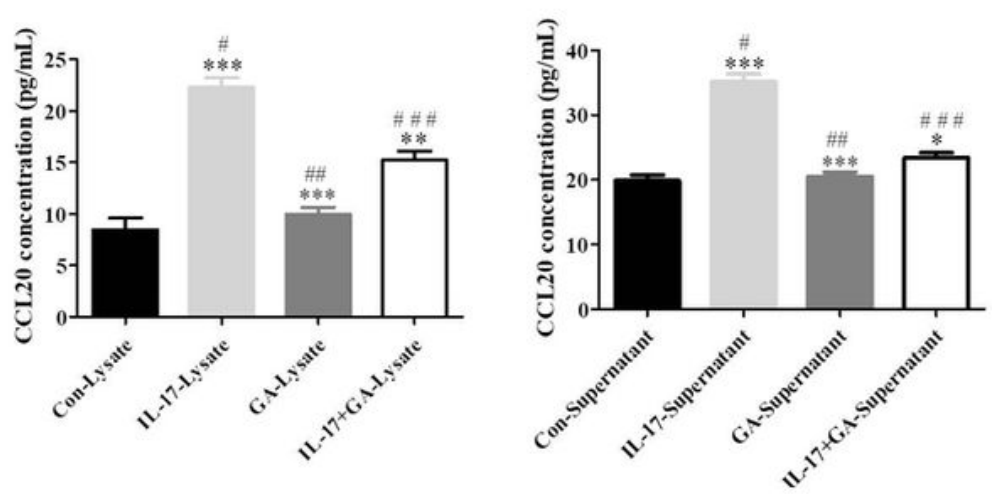

$\mathrm{D}$
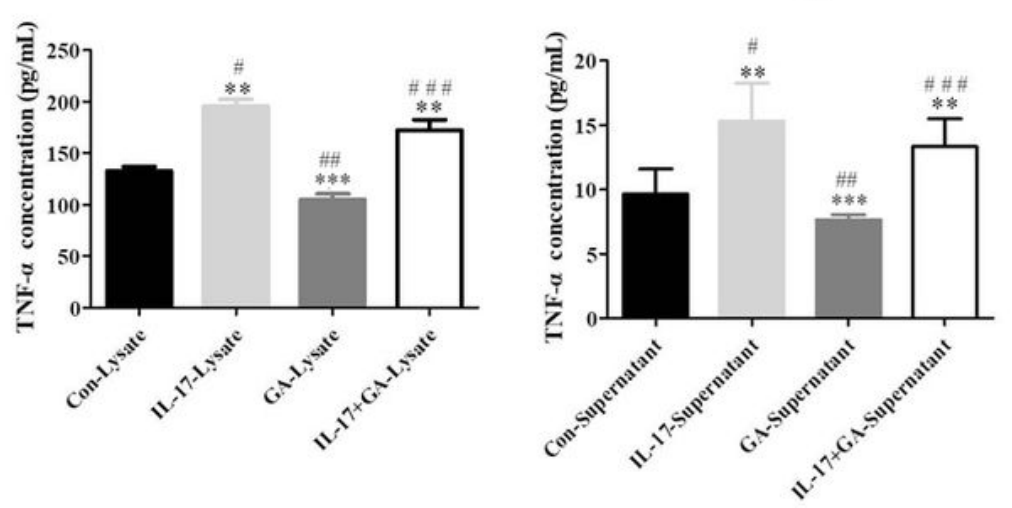

Figure 2 
Glycyrrhizin suppresses cell proliferation and the expression of inflammatory factors in IL-17A-HaCaT cells. (A) The CCK-8 results indicate that IL-17A promoted the proliferation of HaCaT cells, while glycyrrhizin could reverse the proliferation effect of IL-17A on HaCaT cells. (B-D) After treatment with IL17A for 48h, the expression of IL-6 was substantially higher in the lysate and supernatant of HaCaT cells, while glycyrrhizin could reverse the promotion of IL-6 induced by IL-17A (B). Besides, glycyrrhizin has the same regulation of CCL20 (C) and TNF-a (D) as IL-6. NOTES: Data are presented as mean \pm SD $(n=3) . * P$ $<0.05,{ }^{*} \mathrm{P}<0.01$ and ${ }^{* *} \mathrm{P}<0.001$; Con represents wild type HaCaT cells; GA represents glycyrrhizin; IL17 represents IL-17A; \#compared with control group; \#\#compared with IL-17A lysate or supernatant group; \#\#\#compared with GA lysate or supernatant group.
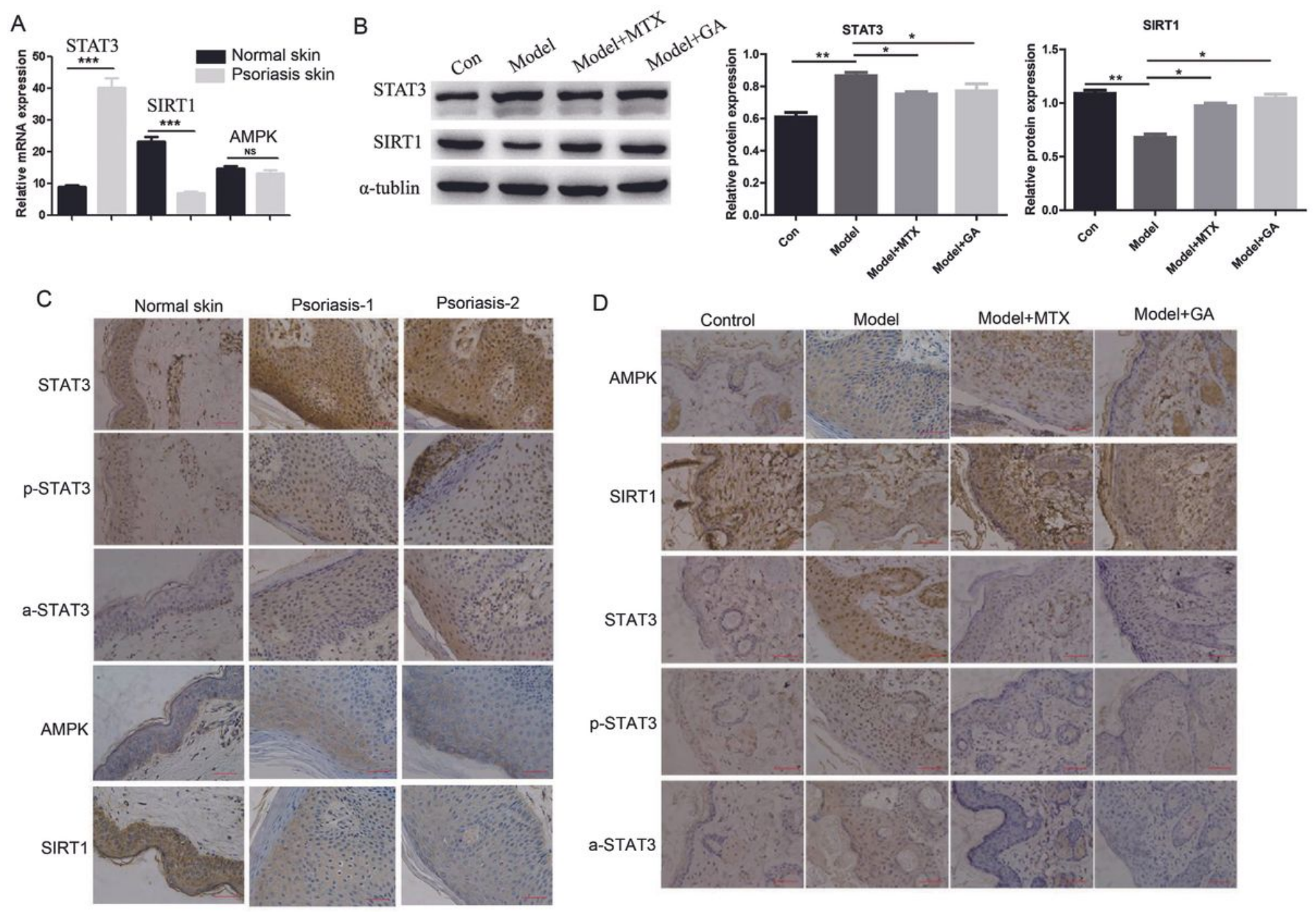

\section{Figure 3}

Glycyrrhizin regulates SIRT1 and STAT3 expression in vivo. (A) RT-PCR results showed that the mRNA expression of STAT3 was up-regulated while SIRT1 was down-regulated in human psoriasis skin tissues $(\mathrm{N}=10)$ than normal skin tissues $(\mathrm{N}=10)$. (B) western blotting results showed that compared with the skin tissues of IMD model, the protein expression of STAT3 was down-regulated while SIRT1 was up-regulated in Model+MTX or Model+GA group. (C) The immunohistochemical results showed that compared with normal subjects, the expression of STAT3 were substantially increased, and the expression of SIRT1 was substantially decreased, while the expression of AMPK was not substantially changed. (D) In IMD, the 
immunohistochemical results showed that when treated with glycyrrhizin, the expression of STAT3 was decreased while the expression of SIRT1 was increased in IMD.

A
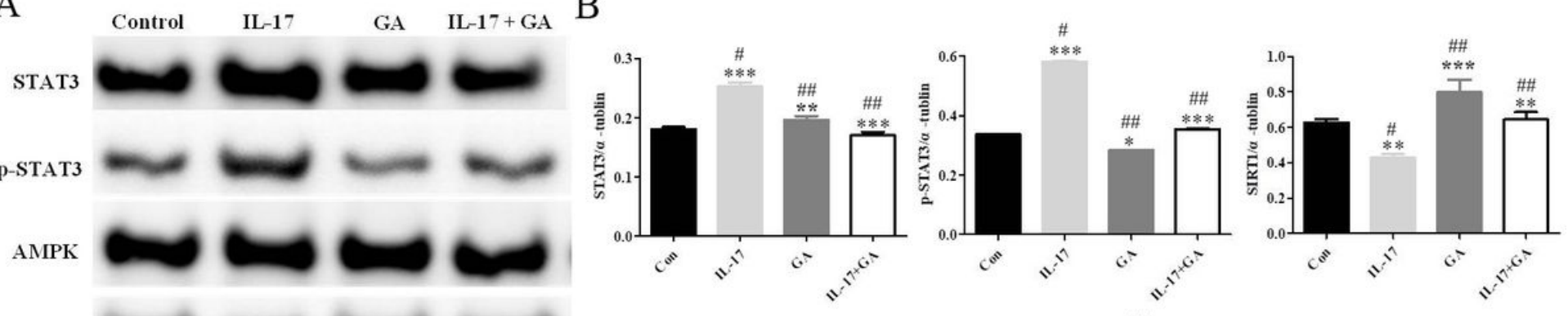

p-AMPK
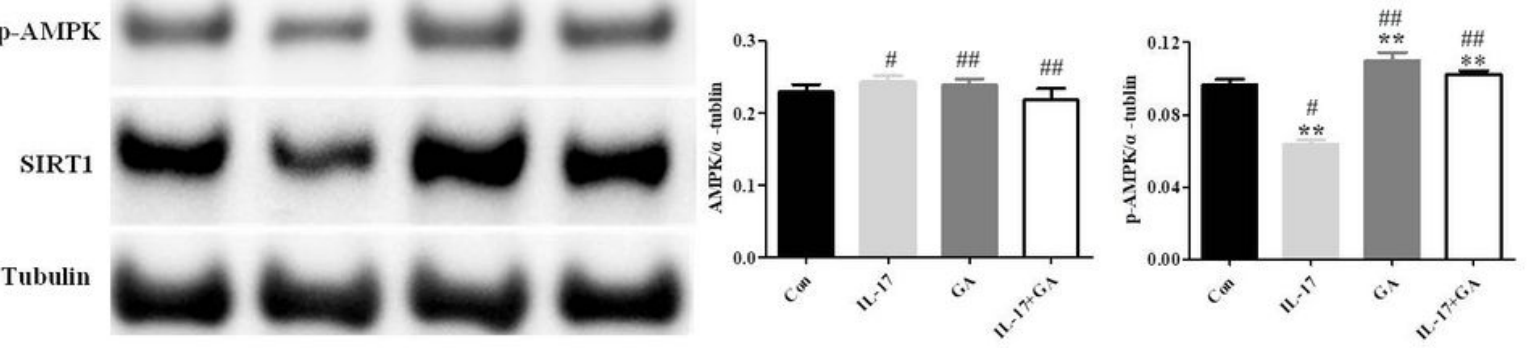

\section{Figure 4}

Glycyrrhizin regulates the expression of SIRT1 and STAT3 in IL-17A-HaCaT cells. (A) In IL-17A-HaCaT cells, glycyrrhizin can substantially reverse the high expression of STAT3 and p-STAT3 while reverse the low expression of SIRT1 induced by IL-17A. (B) Histogram of western blotting results. NOTES: Data are presented as the mean $\pm S D(n=3)$. ${ }^{\star} P<0.05$, ${ }^{\star *} P<0.01$ and $* \star * P<0.001$; Con represents wild type $\mathrm{HaCaT}$ cells; GA represents glycyrrhizin; \#compared with control group; \#\# compared with IL-17A-HaCaT cells; a-tublin is used for internal reference. 


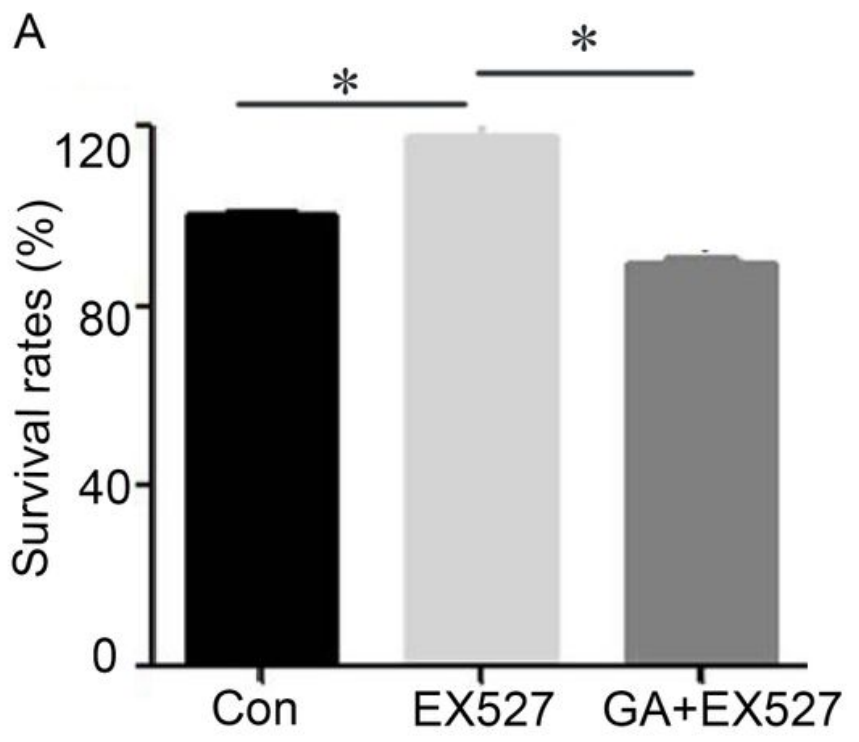

B Con EX527 GA+EX527

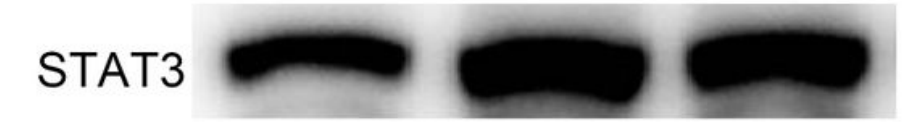
p-STAT3 a-STAT3 a-tubulin
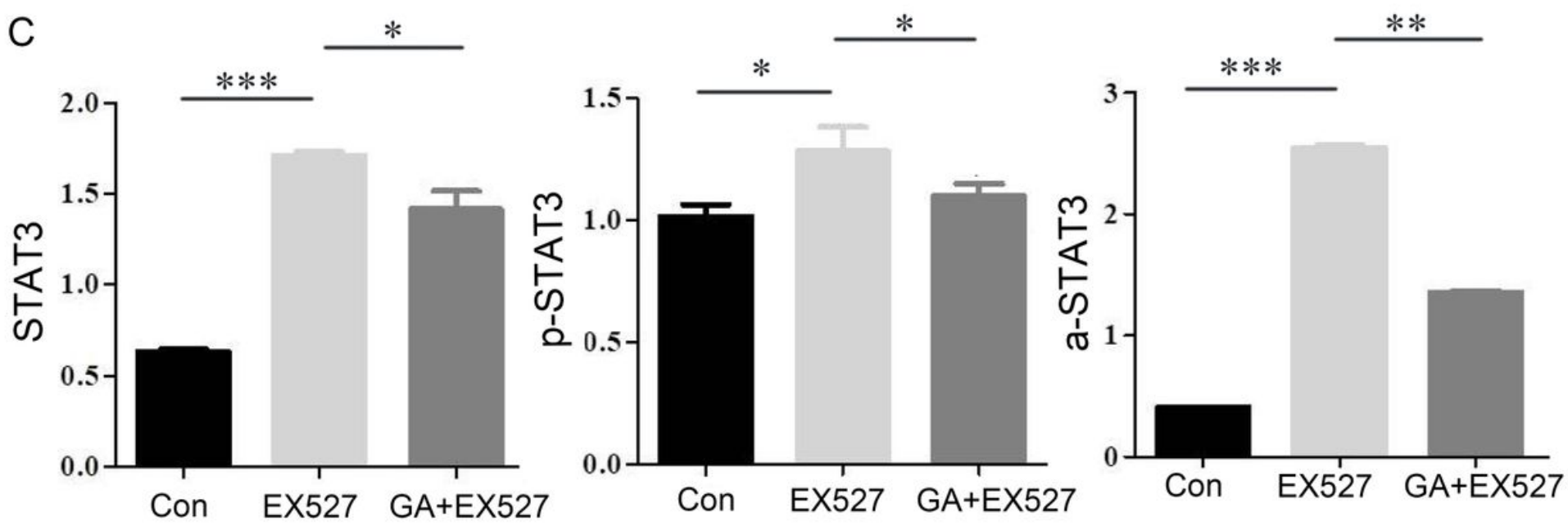

Figure 5

Glycyrrhizin suppresses the expression of STAT3 via SIRT1. (A) The CCK-8 assay was used to determine the effect of EX527 and glycyrrhizin on proliferation of HaCaT cells. (B) The western blotting results show that EX527 promotes the expression of STAT3, p-STAT3, a-STAT3 in IL-17A-HaCaT cells, and glycyrrhizin can reverse the promotion of STAT3, p-STAT3 and a-STAT3 induced by EX527. (C) Histogram of western blotting statistical results. NOTES: Data are presented as the mean $\pm S D(n=3) .{ }^{*} P<0.05$, ${ }^{\star *} P<0.01$ and $\star \star \star \mathrm{P}<0.001$; Con represents wild type HaCaT cells; GA represents glycyrrhizin; a-tublin is used for internal reference. 
A

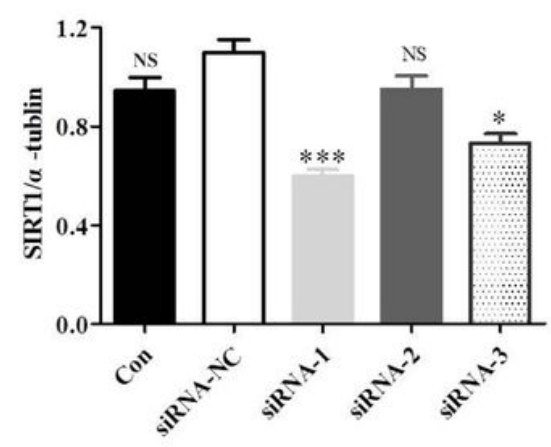

$\mathrm{D}$

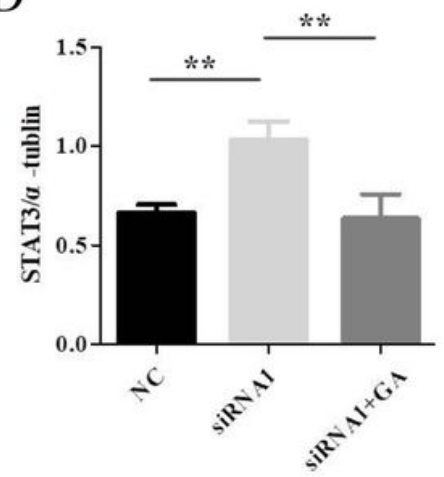

$\mathrm{B}$
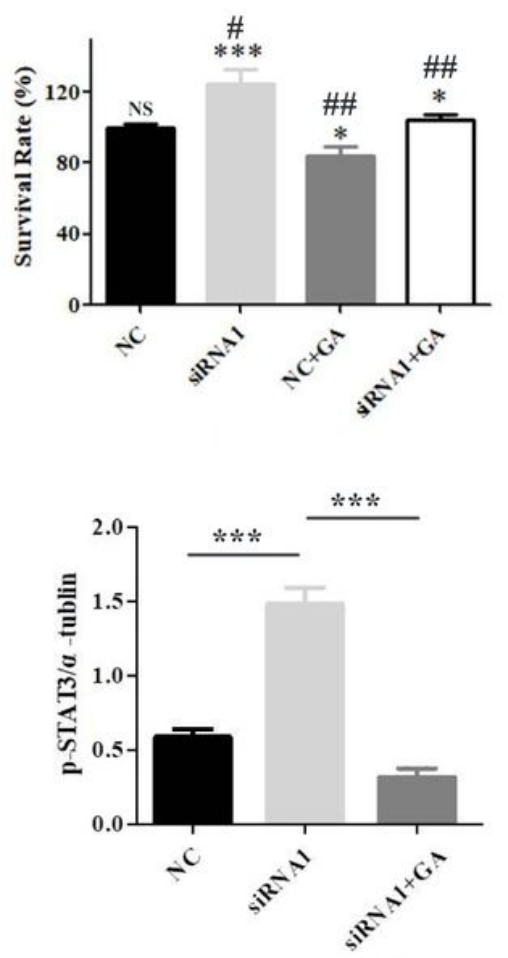

$\mathrm{C}$
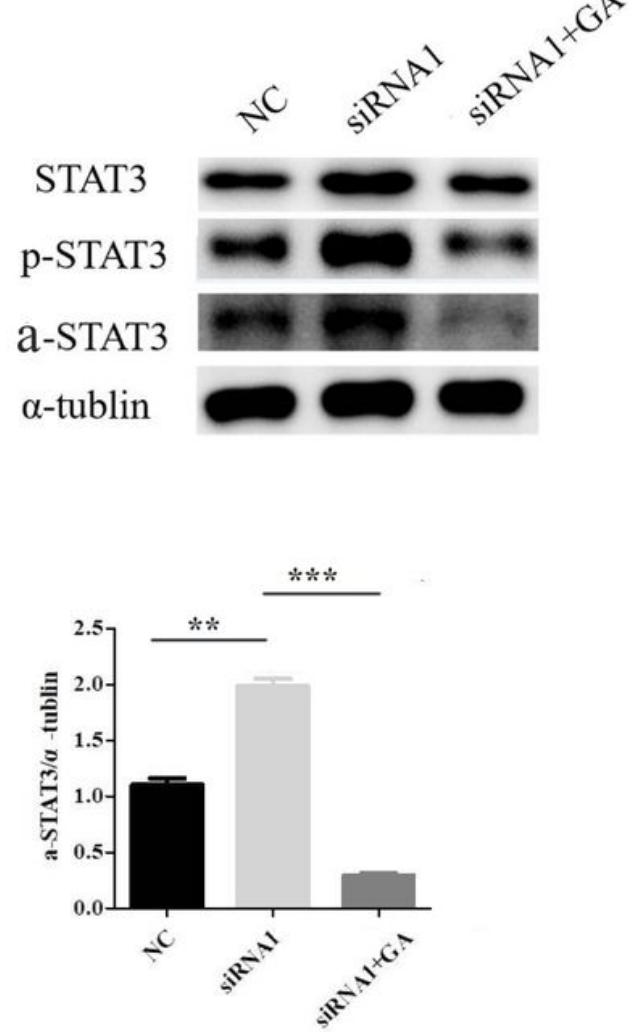

Figure 6

Glycyrrhizin inhibits the expression of STAT3 by up-regulating SIRT1. (A and B): By constructing SIRT1knockdown small interfering RNA $(A)$ and combining with CCK-8 experiments (B), we found that SIRT1knockdown promoted the proliferation of IL-17- HaCaT cells. (C)When knock-down SIRT1 in IL-17- HaCaT cells, glycyrrhizin could reverse the promotion of STAT3, p-STAT3 and a-STAT3 induced by SIRT1 knockdown. (D) Histogram of western blotting statistical results. NOTES: Data are presented as the mean \pm SD $(n=3) .{ }^{*} P<0.05,{ }^{\star} * P<0.01$ and ${ }^{*} * \mathrm{P}<0.001 ; \mathrm{NC}$ represents siRNA-NC transfected HaCaT cells; GA represents glycyrrhizin; \#:compared with NC group; \#\#:compared with siRNA-1 group; a-tublin is used for internal reference. 